Palabra Clave (La Plata)

ISSN: 1853-9912

palabraclave@fahce.unlp.edu.ar

Universidad Nacional de La Plata

Argentina

\title{
Crónicas para una Historia de la Edición y la Lectura en el siglo XIX. La Biblioteca Americana de Alejandro Magariños Cervantes
}

\author{
Valinoti, Beatriz Cecilia \\ Crónicas para una Historia de la Edición y la Lectura en el siglo XIX. La Biblioteca Americana de \\ Alejandro Magariños Cervantes \\ Palabra Clave (La Plata), vol. 9, núm. 2, 2020 \\ Universidad Nacional de La Plata, Argentina \\ Disponible en: http://www.redalyc.org/articulo.oa?id=350562513010 \\ DOI: https://doi.org/10.24215/18539912e083
}

Esta obra está bajo una Licencia Creative Commons Atribución-NoComercial-Compartirlgual 4.0 Internacional. 
Dossier: Catálogos y colecciones editoriales en Latinoamérica: abordajes teóricos, históricos y materiales

\title{
Crónicas para una Historia de la Edición y la Lectura en el siglo XIX. La Biblioteca Americana de Alejandro Magariños Cervantes
}

\author{
Chronicles for a history of editing and reading in the 19th century. The Biblioteca Americana of Alejandro \\ Magariños Cervantes \\ Beatriz Cecilia Valinoti \\ Universidad de Buenos Aires. Facultad de Filosofia y Letras. \\ Instituto de Investigaciones Bibliotecológicas, Argentina \\ DOI: https://doi.org/10.24215/18539912e 083 \\ Redalyc: http://www.redalyc.org/articulo.oa? \\ bvalinoti@filo.uba.ar \\ $\mathrm{id}=350562513010$ \\ Recepción: 05 Febrero 2020 \\ Aprobación: 20 Marzo 2020
}

\section{Resumen:}

La Biblioteca Americana era una colección que, en palabras de Alejandro Magariños Cervantes, estaba destinada a difundir material inédito de los más notables autores americanos. Si bien este proyecto había comenzado en París en 1854, los tomos siguientes fueron publicados en Buenos Aires y Montevideo entre 1858 y 1864. Interesa destacar que en cada libro el editor incluyó algunas páginas que denominó "Crónica de la Biblioteca”, donde presentaba ideas relacionadas con el plan general de la obra, intercambios epistolares, juicios críticos, listas de suscriptores, circuitos de distribución y lugares de comercialización. Todo esto sin dejar de dar cuenta de las dificultades con la impresión, tanto por los retrasos de los autores en entregar las obras como por cuestiones de producción debidas a la falta de papel. A dichos factores se le sumaban los altos costos y las pocas ganancias materiales que se obtenían al llevar adelante esta empresa. De modo que, el objetivo de este artículo es estudiar las crónicas para reconstruir el devenir de un catálogo, comparando los autores y las obras que anuncia publicar Alejandro Magariños Cervantes con el contenido definitivo de la colección. A la vez, se plantea rastrear indicios de prácticas y usos asociados al libro a mediados del siglo XIX.

Palabras Clave: Biblioteca, Literatura americana, Alejandro Magariños Cervantes, Historia de la edición, Historia de la lectura, Siglo XIX.

\section{Abstract:}

The American Library was a collection that, in the words of Alejandro Magariños Cervantes, was intended to disseminate unpublished materials by the most notable American authors. Although this project began in Paris in 1854, the following volumes would be published in Buenos Aires and Montevideo, between 1858 and 1864. It is interesting to note that in each book, the editor included some pages that he called "Chronicle of the Library", where he presented his ideas about the general plan of this work, written correspondence, critical judgments, lists of subscribers, distribution circuits and places of commercialization. He also mentioned difficulties with printing, both due to the delays of authors delivering the works and to production issues due to lack of paper. Additional problems were the high costs and the few material gains obtained by this company. The purpose of this article is to study those chronicles in order to reconstruct the future of the catalog, comparing the authors and the works that Alejandro Magariños Cervantes announces will be published with the definitive content of the collection. At the same time, this article aims to track indications of practices and uses associated with book production in the mid-19th century.

KEYWORDS: Library, Literature of the Americas, Alejandro Magariños Cervantes, Edition History, Reading History, 19th Century.

\section{ENTRAmos EN MATERIA...}

Adentrarse en una biblioteca y perderse en el bosque de sus libros posibilita, entre otras cuestiones, intentar descubrir el mensaje que en sus volúmenes, hojas y palabras puede dar forma a una historia de la edición y de la lectura. En ese sentido, es importante considerar que hoy en día esto es posible no solo al tomar contacto con estas materialidades, sino que también los proyectos de digitalización abren nuevas perspectivas y posibilidades que enriquecen ese recorrido. $Y$ es precisamente un proyecto de esas características llevado adelante por la Biblioteca de la Academia Argentina de Letrasel que, al poner en línea su acervo de obras raras 
y valiosas editadas durante el siglo XIX en Argentina, permitió redescubrir en Esther de Miguel Cané (padre) la Biblioteca Americana. Se trata de una colección que, en palabras de Alejandro Magariños Cervantes, estuvo destinada a difundir material inédito de los más notables autores americanos y convertir la literatura en un agente de civilización y progreso, creando a la vez un nuevo ramo de la industria generador de riqueza.

$\mathrm{Al}$ tomar en consideración ese corpus (con especial atención a aquellas fuentes que indican los títulos a publicarse y las listas de suscriptores) se aplica un método cualitativo e indiciario como el que propone Carlo Ginzburg (2004 y 2010), con el fin de poder reconstruir las dificultades de los editores por conseguir las obras; abordar las maneras de escribir y los modos de leer -junto a las imágenes que representan la lectura-, para posteriormente entender las formas en las que los lectores se apropian de los textos (Jackson, 2001; Parada, 2012; Stoddard, 1985).

Si bien el proyecto de la biblioteca había comenzado en París en 1854 con la publicación de los Estudios Históricos, Politicos y Sociales sobre el Rio de la Plata del propio Magariños Cervantes, los próximos años vieron aparecer -entre Buenos Aires y Montevideo- el resto de los tomos que conformaron la colección. Interesa destacar que, en cada libro, el editor decidió incluir algunas páginas que denominó la "Crónica de la Biblioteca", desde donde compartió ideas relacionadas con el plan general de la obra con sus "amados lectores y escribientes". Asimismo, añadió intercambios epistolares, juicios críticos y listas de suscriptores, sin dejar de dar cuenta de las dificultades con la impresión, tanto por los retrasos de los autores en entregar las obras como por cuestiones de producción debidas a la falta de papel y otros factores. Por un lado, las dificultades de la administración y la distribución; por otro lado, los problemas del trabajo en el taller, a los que se le sumaban los altos costos, la falta de apoyo oficial y las escasas ganancias que se obtenían al llevar adelante esta empresa (Cané, 1858).

De modo que, este artículo tiene como objetivo iniciar el estudio de las crónicas que se publicaron en la Biblioteca América para analizar, desde el punto de vista del editor, la trayectoria del catálogo. Con ello, se pretende reconstruir un episodio de la historia de la edición para aventurarse, a partir de los suscriptores, en el mundo de los lectores de mediados del siglo XIX en Argentina, haciendo propios los deseos de Magariños Cervantes:"serán tal vez de grande importancia para la apreciación de los hombres, y para la futura historia de la literatura americana. Entramos en materia". ${ }^{1}$

\section{Breve SEMblanza DEL EDITOR}

Magariños Cervantes ${ }^{2}$ nació en 1825 , en la ciudad de Montevideo. Fue poeta, narrador, ensayista y periodista, a la vez que abogado, fiscal, docente (catedrático de Derecho Natural), cónsul en Buenos Aires, ministro, senador y rector de la Universidad de la República (1878-1880). El editor vivió en Río de Janeiro y en varias ciudades europeas, donde publicó la mayor parte de su obra, como la Revista Española de Ambos Mundos- impresa por el establecimiento tipográfico de Mellado- que apareció simultáneamente en Madrid y París entre 1853-1855 (Hartzenbusch e Hiriart, 1894). En esta última ciudad fue donde Magariños Cervantes dio inicio a una colección de escritores americanos, llamada Biblioteca Americana, en la que se pensaba incluir obras históricas, científicas, filosóficas y literarias (novelas, leyendas, poesías). Así, el propósito era estimular el interés de los lectores con una oferta tentadora de autores (Auza, 2002).

En 1854, Magariños Cervantes publicó Estudios históricos, políticos y sociales sobre el Río de la Plata, primer libro de investigación histórica sobre Uruguay que no solo introduce al gaucho de manera reivindicativa, sino que lo incorpora en la tradición literaria uruguaya a la vez que crea una épica nacional con el canto a lo nativo. Esas cuestiones, en palabras de Ángel Rama, lo convirtieron en el escritor que modela e institucionaliza el romanticismo con su Álbum de poesías (1878), acuñando el prototipo de la novela histórica con Caramurú (1850) y la leyenda poética con Celiar (1852) (Coll, 2018).

Reconocido por sus coetáneos (Figura 1), Juan Pivel Devoto (1963) rescata, como elementos que distinguen su personalidad, la concepción americanista de sus temas, la honradez con que trabajó para dar a 
las letras un acento nacional y el hecho de haber divulgado en un escenario europeo los rasgos más salientes de la vida de estos pueblos. No obstante, la Biblioteca Americana se constituye como la muestra concreta en donde estas cuestiones se presentan de forma evidente.

Figura 1. Dr. Alejandro Magariños Cervantes

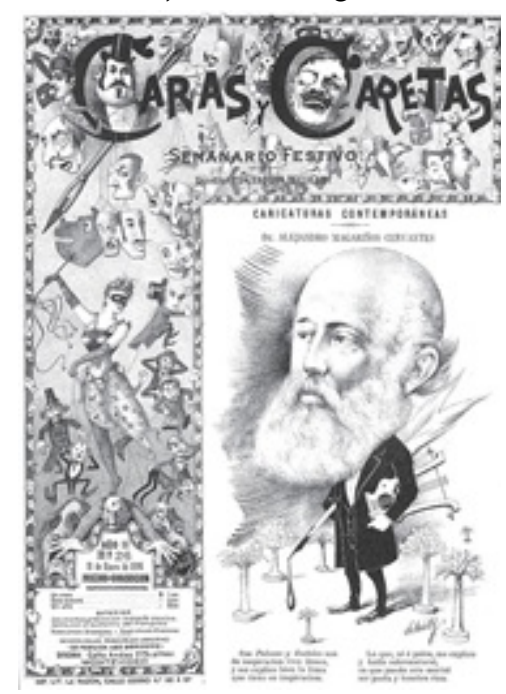

Fuente: Portada de Caras y Caretas $N^{\circ} 26$, Montevideo (Enero de 1891).

\section{LA iDEA DE una Biblioteca Americana}

La propuesta de editar una Biblioteca Americana no fue una novedad. Magariños Cervantes pudo haber encontrado un antecedente en el proyecto iniciado en Londres en 1823 por Andrés Bello y Juan García del Río quienes, al presentárselo a Antonio José Irisarri, Ministro de Chile en Londres, afirmaron:

La general necesidad que tienen los nuevos países americanos de papeles útiles que contribuyan a la ilustración de los ciudadanos en ramos tan interesantes como las ciencias, las artes y las letras, nos permite esperar un eficaz apoyo para cumplir con un propósito a todas luces elevado y noble en el cual no hay como bien puede Ud. apreciarlo, intenciones de lucro (Bello, 1984, pp. 127-128).

En el "Prospecto" de la obra se ampliaban las intenciones que guiaban este emprendimiento editorial, siendo la más importante la pretensión de presentar al pueblo americano las riquezas intelectuales de los pasados siglos, a fin de servir como preparación para el futuro. Esta tarea fue definida como enciclopédica, ya que Bello y del Río entendían que transmitir los tesoros del ingenio y del trabajo americano permitiría remover la ignorancia, lo que se transformaba en un signo de libertad. La empresa quedaba delineada como una obra de puro patriotismo ya que los redactores no pretendían otra ganancia que la de servir a sus conciudadanos. Sin preferencias por algún pueblo, se la escribía para todos: colombianos, argentinos, peruanos o chilenos; en tal sentido se proclamaba esa biblioteca como americana (Biblioteca Americana, 1823).

Allí también se caracterizaba el contenido de las tres secciones que compondrían cada volumen: Humanidades y Artes Liberales; Ciencias Matemáticas y Físicas, con sus aplicaciones y finalmente Ideología, Moral e Historia. Se anunciaba además que todos los tomos tendrían esta distribución, de modo que cada número podía componer un libro bastante voluminoso, que además se adornaría con láminas a color (Figuras 2 y 3$)$. 
Figura 2. La Biblioteca Americana, o, Miscelánea de Literatura, Artes y Ciencias. 1823

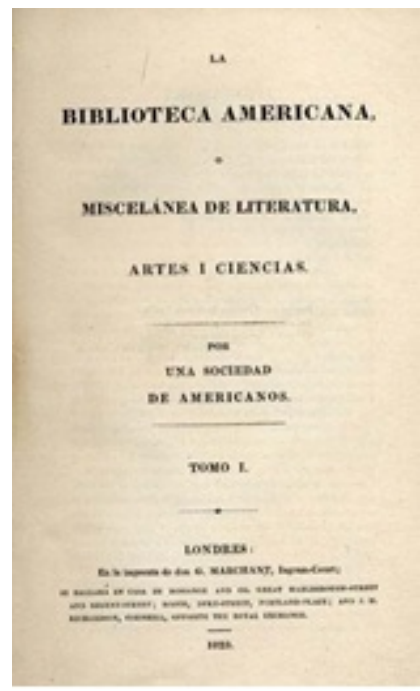

Figura 3. Ilustración para alocución a la poesía en que se introducen alabanzas de los pueblos e individuos americanos. Cooke, George / Corbould, Henry. 1823

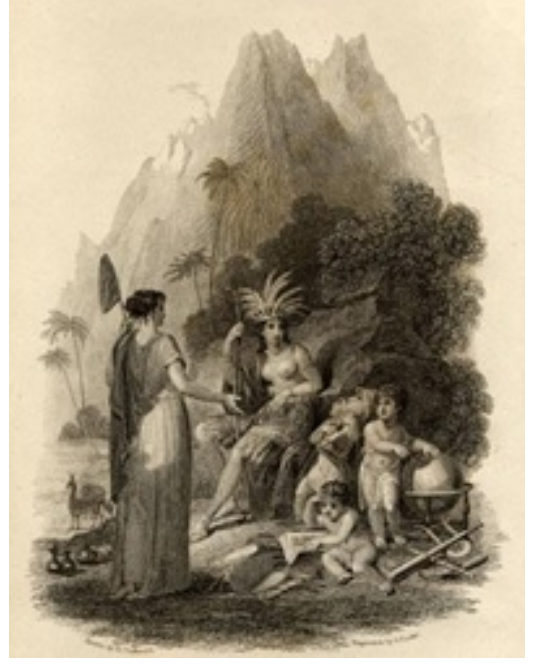

El 16 de abril de 1823 apareció la Biblioteca Americana o Miscelánea de Literatura, artes iciencias, publicada por una Sociedad de Americanos entre los que figuraban además del propio Bello, ${ }^{3}$ García del Río, Luis López Méndez, Pedro Creutzer, don P. Cortes y otra persona que firmaba con las iniciales A. G. M. Esta publicación fue impresa en Londres, en el establecimiento de don G. Marchant, Ingram-Court, y circuló un volumen completo con 474 páginas.

Como editor, Bello complementó ese proyecto con la publicación de ensayos que consideraba de utilidad para las nuevas repúblicas. También extractaba, reseñaba y traducía artículos que provenían de publicaciones inglesas y francesas, siendo de ese modo un iniciador y generador del diálogo de la literatura americana en el plano universal (Jaksic Andrade, 2001).

Sin embargo, uno de los problemas principales de la biblioteca eran los costos, ya que una edición lujosa e ilustrada dependía del patrocinio de los pueblos y de los gobiernos americanos (Biblioteca Americana, 1823, p. VI). Tal es así que, después de remitir la mayoría de los ejemplares a América, las dificultades en las comunicaciones con las nuevas repúblicas impidieron hacer efectivo el cobro de las suscripciones y se vieron imposibilitados de cumplir con nuevos pedidos (Amunátegui Aldunate, 1882). Si bien los editores se habían 
propuesto publicar un cuaderno cada vez que se lo permitieran las ocupaciones de los redactores convocados, los problemas financieros frustraron la continuidad del proyecto.

\section{Crónica de UNA BiBlioteca}

En julio de 1858, Magariños Cervantes reconoció que había vuelto a su antigua empresa de formar una Biblioteca Americana. Así, Juan María Gutiérrez recuerda que este pensamiento

bajo diversas formas bulle hace muchos años en la cabeza de varios amigos del progreso intelectual de nuestro continente. Su primer germen brotó... con las plumas de Bello y García del Río... el hecho es que... la Biblioteca, tan conocida por los estudiosos, quedo a medio camino...(Cané, 1858, pp. 7-8).

El escritor advierte que lo que ocurrió con aquella publicación fue una lección que no debería ser olvidada (Cané, 1858). El tiempo había pasado desde aquella iniciativa y, aunque Magariños Cervantes afirmaba sobrarle fe y perseverancia, entendía que los desafíos seguían siendo muchos. Algunos retos eran similares a los que debieron hacer frente Bello y García del Río, pero otros nuevos aparecían en su horizonte. Por un lado, había que tener presente cómo financiar el proyecto para hacerlo viable. Por otro, debía mantener la calidad de la colección y contar con suficientes obras para no retrasar la publicación. A su vez, tenía que atender a la falta de papel, los escasos talleres de impresión, la ausencia de redes de distribución, las pocas librerías, los problemas y tardanzas en el cobro de las suscripciones, sin dejar de considerar las demoras en la entrega de las correcciones y el cuidado de la edición de un catálogo (Magariños Cervantes, 1858b).

Si bien se reconoce el proyecto de Bello como antecedente, hay que tomar en cuenta que durante el siglo XIX en Hispanoamérica, un conjunto de antologías acompañaron a estas iniciativas. En palabras de Estuardo Núñez (1959) estos trabajos cobraron importancia ya que intentaron afirmar la autonomía espiritual de los nuevos pueblos de este continente, pretendiendo dar cuenta de una cierta idea de unidad entre las élites intelectuales de estos países, sobre todo los que reconocían un mismo origen y tradición. Así se editaron tres obras con el mismo título: América Poética. La primera, fue publicada en Valparaíso por Juan María Gutiérrez (1846), otra compilada por Rafael María de Mendive y José de Jesús Q. García (1854-1856) y una tercera elaborada por José Domingo Cortés (1875). A estas obras pueden sumársele Flores del Nuevo Mundo de Manuel Nicolás Corpancho (1863), América Literaria de Francisco Lagomaggiore (1890), los dos volúmenes de Literatura Americana de Martín Coronado (1885) y Joyas poéticas americanas. Colección de poesias escogidas originales de autores nacidos en América de Carlos Romagosa (1897).

Magariños Cervantes, siguiendo las mismas aspiraciones que guiaban a los escritores de la generación romántica, en una "Epístola Programa" (1854) anunciaba la Biblioteca Americana, un proyecto que creaba en Europa una tribuna para el pensamiento americano que se concretaba en libros (Pivel Devoto, 1963). Con la colección en marcha se informaba que cada tomo contendría unas 300 páginas, que sería de una forma y tamaño similar a los Estudios sobre el Rio de la Plata, en buen papel y esmerada impresión. Se la presentaba como una Biblioteca por la diversidad de autores, una Enciclopedia por la homogeneidad y enlace de las materias a la vez que una Revista, es decir un órgano autorizado de la literatura americana por la "altura y el nombre de sus redactores, por la originalidad, por el colorido local, reflejo de muestra naturaleza, de nuestras costumbres, de nuestra vida política y social; y finalmente por la tendencia fecunda, democrática y civilizadora de sus páginas" (Magariños Cervantes, 1858a, p. 302).

A partir del tomo II, Magariños Cervantes anunció su intención de destinar algunas páginas de cada ejemplar para presentar artículos, juicios críticos, comentarios en la prensa, cartas e indicaciones referidas a las obras que se iban publicando, aclarando que esta no era una cuestión original, ya que propuestas similares se encontraban en publicaciones europeas o en la América Poética de Gutiérrez. ${ }^{4}$ Fueron los contenidos de dicha "Crónica de una Biblioteca" los que permitieron reconstruir el devenir de un catálogo, al comparar los 
autores y las obras que anunciaba publicar, con el contenido definitivo de la colección, a la vez que rastreaba indicios de prácticas y usos asociados al libro a mediados del siglo XIX.

\section{Autores y obras para un CATÁlogo}

Existen múltiples cuestiones que pueden ser analizadas a partir de esas páginas. Sin embargo, una de las primeras que merecen atención gira en torno a la propuesta de obras y autores (Tabla 1). Si los suscriptores eran financieramente importantes para el proyecto, se suponía que éstos querrían saber con anticipación las obras a publicarse. Este es uno de los primeros aspectos a los que se abocó Magariños Cervantes al volver de Europa. Luego de haber aparecido el primer tomo, el editor informó alguno de los títulos que formaron parte de la colección. Asimismo, señalaba que unos trabajos ya estaban terminados, mientras que algunos de los autores convocados -comprometidos a escribir obras nuevas- le solicitaron tiempo para preparar los manuscritos; de modo que se había resuelto reimprimir algunas otras obras para no paralizar las entregas (Magariños Cervantes, 1858a). En vistas de ello, una veintena de títulos le dieron forma a la primera propuesta del catálogo, un plan ambicioso que se proyectaba de largo plazo. 
Tabla 1. Primera propuesta de catálogo de la Biblioteca Americana

\begin{tabular}{|c|c|c|}
\hline Autor & Título & Comentarios \\
\hline Hilario Ascasubi & Cuentos y trovas de la pampa & Sin registro de su publicación \\
\hline Francisco Bilbao & El gobierno de la libertad & $\begin{array}{l}\text { Había aparecido por la Imprenta del } \\
\text { Comercio, Lima en } 1855 .\end{array}$ \\
\hline Miguel Cané & Laura, Esther, novelas Originales & $\begin{array}{l}\text { Solo Esther será publicada enla } \\
\text { Biblioteca Americana, en } 1858\end{array}$ \\
\hline Luis Domínguez & Un tomo de poesias & Sin registro de su publicación \\
\hline Heraclio Fajardo & Luz del Alba, leyenda poética & Sin registro de su publicación \\
\hline Félix Frías & Estudios morales y políticos & Sin registro de su publicación \\
\hline Manuel R. García & $\begin{array}{l}\text { Documentos inéditos para la Historia del Río } \\
\text { de la Plata }\end{array}$ & Sin registro de su publicación \\
\hline $\begin{array}{l}\text { Juan Carlos Gómez } \\
\text { (Uruguayo) }\end{array}$ & Uno tomo de poesias & Sin registro de su publicación \\
\hline General D. Tomas Iriarte & Recuerdos Históricos: 1817 & Sin registro de su publicación \\
\hline José Mármol & Después del Triunfo & Sin registro de su publicación \\
\hline Bartolomé Mitre & Arengas & $\begin{array}{l}\text { Será publicado por la Imprenta } \\
\text { Especial para Obras de Pablo Coni, } \\
\text { en Buenos Aires, } 1875\end{array}$ \\
\hline Miguel Navarro Viola & Meditaciones religiosas y filosóficas & Sin registro de su publicación \\
\hline Martin Avelino Piñero & El púlpito argentino & Sin registro de su publicación \\
\hline Domingo F. Sarmiento & Palermo y Santos Lugares & Sin registro de su publicación \\
\hline Marcos Sastre & Cuadros e impresiones del Paraná & $\begin{array}{l}\text { Publicado por la Biblioteca } \\
\text { Americana en } 1858\end{array}$ \\
\hline Carlos Tejedor & Curso de derecho criminal & $\begin{array}{l}\text { Será publicado por la imprenta Joly, } \\
\text { en Buenos Aires, } 1860 \text {. }\end{array}$ \\
\hline Héctor F. Varela & Ojeada sobre el Paraguay & Sin registro de su publicación \\
\hline Dalmasio Vélez-Sarsfield & Banco de crédito en Buenos Aires & Sin registro de su publicación \\
\hline Carlos Maria de Viel-Castel & $\begin{array}{l}\text { Monografia de la prensa bonaerense,ó } \\
\text { reseña Bibliográfica de todos los periódicos } \\
\text { que se han publicado en Buenos Aires } \\
\text { desde } 1810 \text { hasta el presente. }\end{array}$ & Sin registro de su publicación \\
\hline
\end{tabular}

Fuente: elaboración propia.

La característica más sobresaliente de la lista es el hecho de que solo una obra de Miguel Cané y otra de Marcos Sastre formaron parte de la Biblioteca Americana, mientras que la mayoría de los títulos allí consignados no llegaron a la imprenta, a pesar de tratarse de una publicación importante -en palabras de Magariños Cervantes- por la cantidad de suscriptores y lectores con los que contaba. Sin dudas, este era un factor necesario pero no suficiente para vencer los contratiempos y demoras que se le presentaban a este proyecto e impedían su concreción, aunque en otros casos pudieron ser editados por otros talleres.

Sirva como ejemplo las Arengas de Bartolomé Mitre, lo cual permite comprobar que ni aun disponiendo de los textos era fácil publicar. Este caso se corresponde con una de las maneras en que se le daba forma a los libros, es decir con la colección de artículos publicados en la prensa, recopilación de discursos u otras disertaciones y, según consta en la segunda edición de la obra, fue un proyecto que recién se había logrado concretar en 1874 (Mitre, 1889). En relación a esta forma de componer un libro guarda interés el caso de las Poesías de Luis Domínguez. Al respecto, Magariños Cervantes explicaba al instar amigablemente al autor a que le entregase la 
colección de poemas que le había ofrecido publicar: "como nos sucede con harta frecuencia, nos encontramos con que en todo había pensado el autor menos en tener prontos los originales para el momento en que se los pidiésemos". Domínguez argumentó que le había sido imposible buscar sus poesías diseminadas en varios periódicos, olvidadas entre sus papeles y hacerlas copiar para entregar al editor (Varela, 1859).

Además de la lista, de acuerdo con un anuncio que se publica en el mismo tomo II de la Biblioteca, cinco obras ya se encontraban en prensa esperando aparecer, una por mes, durante el segundo semestre de 1858. No obstante, este plan tampoco logro realizarse, ya que fueron otras las obras que finalmente terminaron publicándose. Nuevamente la pregunta ipor qué no se pudo cumplir con una propuesta que parecía encaminada a realizarse?

$\mathrm{Al}$ respecto, llama la atención lo que sucedió con Laura de Cané (p). En Horas de melancolía (Figura 4) se había anunciado que la novela ya estaba en la imprenta próxima a salir, programándose la entrega para septiembre de ese año. Sin embargo, dos tomos más adelante, en el cuarto, Magariños Cervantes afirmaba que había sostenido una descomunal guerrilla con el autor para que terminara cuanto antes la copia del manuscrito de Esther, corrigiese pronto las pruebas y satisficiera la voracidad de la imprenta, a la vez que anticipaba que los borradores de Laura estaban en la cartera del autor (Cané, 1858). ¿Era esto una estrategia editorial debido al éxito de las obras del escritor?, ¿implicaba desinformación del editor? o ¿falsas promesas del autor? Pueden esbozarse algunas respuestas a las cuestiones anteriormente enunciadas, aunque una parece primar (y aparece en varias oportunidades): se escribía y se editaba restándole tiempo a las actividades cotidianas, entre problemas domésticos y sin obtener ganancias por estas tareas. En este caso en particular, lo cierto fue que después de la muerte de Cané (p), en 1863 se publicó en el Correo del Domingo un fragmento de la obra aclarándose que ese debería ser considerado el primer capítulo de una novela que pensaba concluir, aun cuando sus amigos (o algunos de sus editores) la pensaban terminada o la hayan anunciado en la imprenta (Curia, 2012).

Figura 4. Obras a editarse

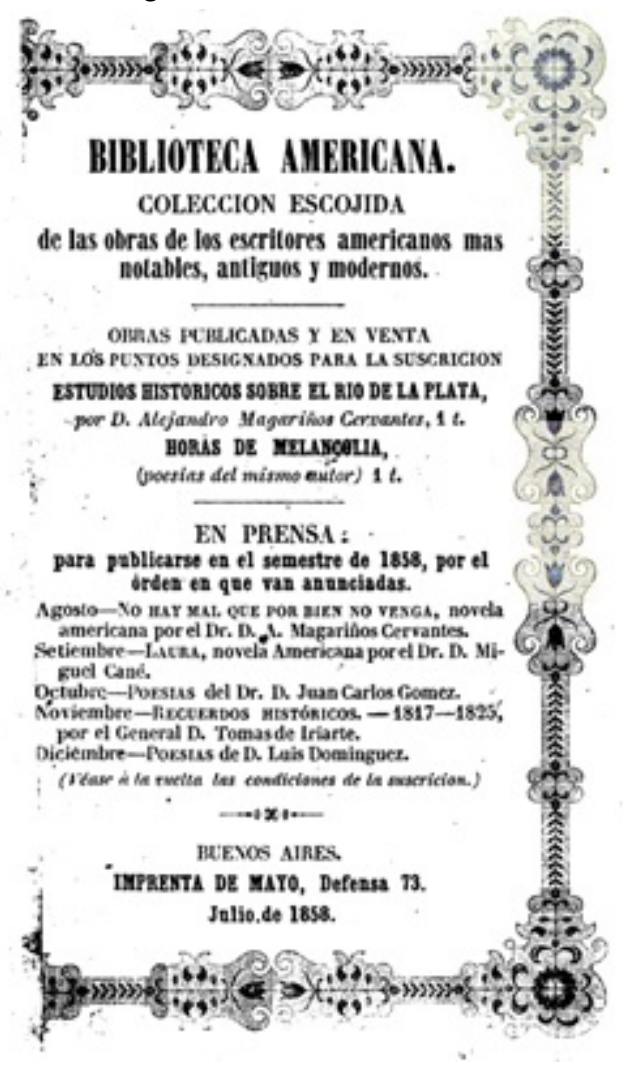

Fuente: Horas de melancolía. Tomo II. 
Otra cuestión que destacaba al volver a la primera lista es que, a pesar de anunciarse como americana, en su mayoría los escritores que se presentaban eran de Buenos Aires. Entonces, ¿era la primera parte de un proyecto más amplio?, ¿se estaba esperando la respuesta del público para avanzar con otras obras y autores?, ¿resultaba preciso consolidar otros circuitos de producción y distribución? Estas son cuestiones muy complejas que ponen en evidencia las múltiples dificultades que impedían concretar las ideas y convertirlas en un libro, independientemente de que se trataran de obras nuevas, reediciones o una recopilación de papeles que ya se encontraban escritos.

Firme en su empeño de editar la colección, en el tomo III Magariños Cervantes anunciaba otra propuesta de catálogo mucho más ambiciosa (Figura 5).

Figura 5. Propuesta de obras y autores.
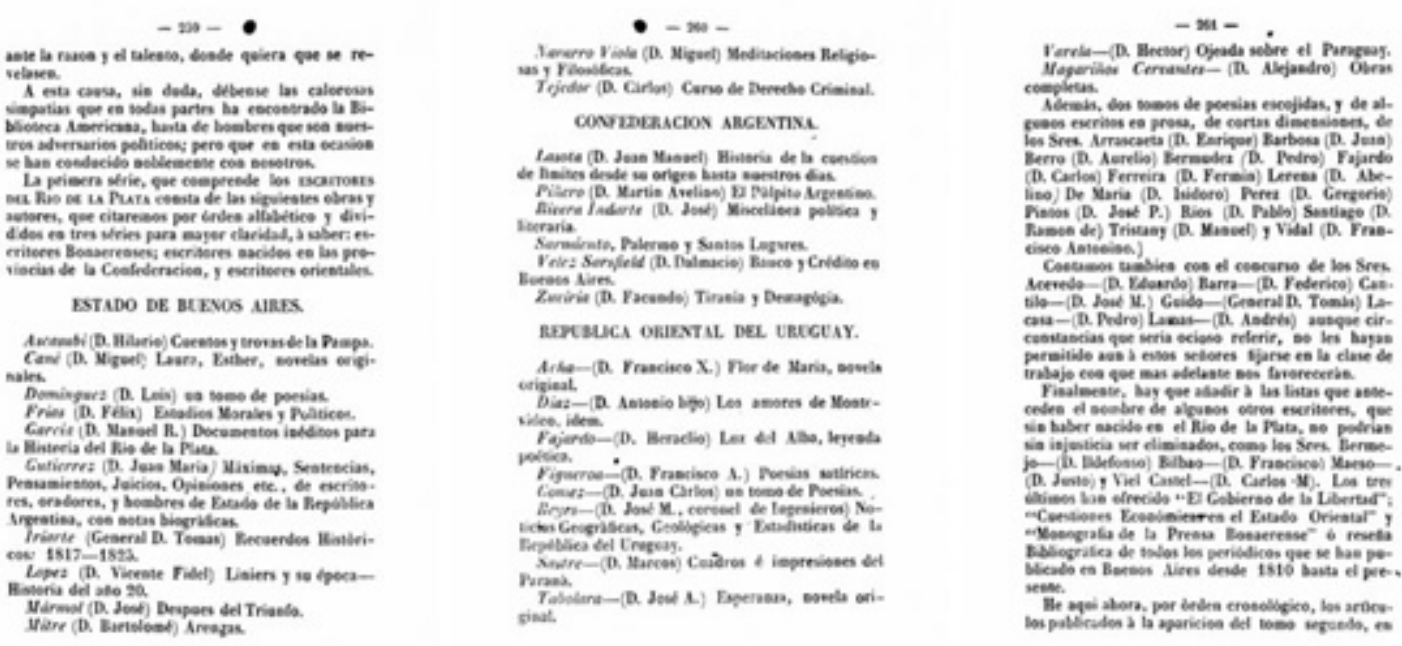

Fuente: No hay mal que por bien no venga Tomo III.

Distribuidos en tres zonas la Biblioteca Americana ofrecía obras de escritores del Estado de Buenos Aires; escritores nacidos en las provincias de la Confederación y aparecieron los escritores orientales, junto a otros que, sin ser nacidos en el Río de la Plata, no podrían ser eliminados de la colección (aunque dos de ellos, Francisco Bilbao y Carlos María de Viel-Castel, ya estaban incluidos en la primera lista aparecida en el tomo II). Esta selección, que habla más bien de una biblioteca rioplatense que americana, atendía, según el editor, a una fase inicial del proyecto pero desplegaba otro elemento novedoso: se presentaba como una colección que reunía distintas corrientes de pensamiento. En ese sentido, eran incluidos hombres que, a pesar de ser adversarios políticos, habían entendido que en esta ocasión debían conducirse noblemente respondiendo al ideal de servir a la unión nacional (Auza, 2002; Magariños Cervantes, 1858b).

Más allá de las buenas voluntades, tales comopublicar un volumen por mes, aumentar los suscriptores para poder financiar una imprenta donde publicar la Biblioteca o lograr la predisposición de los autores que habían ofrecido más de treinta títulos, Magariños Cervantes entendía que había algunos problemas determinantes que, al desarrollarse en un contexto político complejo, terminarían por definir el catálogo de la colección. Estos inconvenientes fueron no contar con la fortuna necesaria para subordinar a sus necesidades el trabajo de las imprentas ni con los medios para forzar al público lector a suscribirse (Gutiérrez, 1859). Principalmente estas cuestiones serán las que terminarán afectando la periodicidad y la cantidad de los volúmenes publicados.

Así, tras el anuncio del inicio de la colección, el primer tomo fue editado, en París en 1854 por la imprenta de Blondeau.

Tomo I. Estudios históricos politicos y sociales sobre el Rio de la Plata de Alejandro Magariños Cervantes. 1 tomo. (415 páginas) 
Luego se trasladó a Buenos Aires y publico por la Imprenta de Mayo, en 1858, cuatro volúmenes más:

Tomo II. Horas de melancolía de Magariños Cervantes (Poesías), 1 tomo. (316 páginas).

Tomo III. No hay mal que por bien no venga de Magariños Cervantes. Novela original, 1 tomo. (308 páginas).

Tomo IV. Esther. La familia Sconner de Cané (p). Novela original, 1 tomo. (296 páginas).

Tomo V. El tempe argentino. Cuadros e impresiones del Paraná de Sastre. 1 tomo. (306 páginas).

Sin ignorar que fueron los suscriptores los que sostuvieron la publicación de la colección, y debido a todas las dificultades a las que se debió enfrentar, no solo se volvieron a destacar los infortunios domésticos o las ocupaciones cotidianas de los autores que impedían cumplir con la entrega de las obras prometidas, hasta los siempre presentes problemas con el papel o las imprentas sino que también se cambió de estrategia. En efecto, se decidió no anunciar los próximos títulos a fin de poder reemplazar las obras que faltaban con otras de la numerosa lista con la que se contaba (Magariños Cervantes, 1858b). A pesar de esta modificación tampoco se pudo seguir el plan original de editar un libro por mes y el próximo tomo apareció al año siguiente, en 1859:

Tomo VI. Pensamientos, máximas y sentencias de escritores, oradores y hombres de estado de la República Argentina de Gutiérrez. 1 tomo (316 páginas).

Dado que la Imprenta de Mayo, que era donde se publicaba la Biblioteca Americana, se veía imposibilitada de cumplir su compromiso, ya que tenía que atender trabajos pautados con otras publicaciones que ocupaban las prensas (como La Religión, La espada de Lavalle, La Nueva Generación, La Historia de Belgrano, La Revista Española, Colección de leyes y decretos, El Museo Literario, El Clamor de los Libres y otras hojas sueltas), se cambia de taller de impresión con la intención de asegurar la regularidad en la aparición de los volúmenes. La colección se editó entonces por la Imprenta del Orden y se publicó ese mismo año:

Tomo VIII. Escritos politicos, económicos y literarios del doctor... de Florencio Varela. Precedidos de su biografía, por D. Luis L. Domínguez. 1 tomo. (332 páginas).

Por entonces, las referidas crónicasse transformaron para evitar la monotonía, así se modificó su contenido suprimiendo los artículos apologéticos y las listas de suscriptores. Se puso interés únicamente en las obras publicadas -como simple aviso que convenía reproducir para facilitar la venta- señalándose que el primero, tercero y quinto tomo se habían agotado completamente, mientras que los otros cuatro se vendían en los puntos designados para la suscripción (Varela, 1858).

Posteriormente, apareció otra cuestión a considerar que eran los problemas con el mundo del taller de imprenta y, desde su perspectiva, Magariños Cervantes comenzó a esbozar algunas respuestas. Entendía que parte de las dificultades se originaron en torno al papel, que a veces no se lo encontraba a ningún precio; aunque señalaba que las mayores complicaciones se relacionaban con el trabajo desarrollado, ya sea por parte de los cajistas, que con pretextos aplazaban la impresión de un pliego hasta tres o cuatro días, o por los encuadernadores, que demoraban la terminación del libro. Según él, no había forma de que fueran doblando los pliegos a medida que los recibían, y los dejaban acumular, para terminar haciendo todo tarde y mal (Gutiérrez, 1859, p. 6 y 7). Estos problemas, sumados al hecho de cambiar de taller, explicaban que el tomo delos Escritos politicos, económicos y literarios de Florencio Varela, que llevaba el número VIII, se haya puesto en circulación quedando en blanco el número VII.

Más allá de que las imprentas estaban recargadas de trabajo y que el papel fuera escaso, estas contrariedades debían ser superadas, aunque el editor señalaba que esto sería raro y excepcional. Sus intenciones eran cumplir con los compromisos pendientes, aunque esto era materialmente imposible; las decisiones estaban pensadas para que la variedad de obras a publicarse los satisfagan (Gutiérrez, 1859). Finalmente el tomo VII apareció en 1860:

Tomo VII. Apuntes biográficos de escritores, oradores y hombres de estado de la República Argentina de Gutiérrez. 1 tomo. (291 páginas).

Si bien en 1864 se había anunciado el fin del emprendimiento, se publicaron los últimos dos tomos por la imprenta Tipográfica a Vapor de Montevideo unificados en un solo ejemplar: 
Tomo IX-X. Brisas del Plata de Magariños Cervantes. (256 páginas).

Aquí, al dirigirse a los suscriptores, el editor les solicitaba que acepten esta publicación como parte de la colección aunque se había modificado el formato (condicionado, una vez más, por la falta de papel), reunido en un solo tomo lo que debía presentarse en dos y aumentado el precio de la suscripción (Magariños Cervantes, 1864). Interesa señalar que este pedido remite a unas ideas presentes desde el comienzo del proyecto: este tipo de propuestas no prosperaban con esfuerzos individuales, ni aunque los escritores obsequiaran sus obras. Tampoco se lograban los resultados esperados por las subvenciones oficiales o la suscripción de un reducido grupo de amigos; solo se fundaban y echaban raíces indestructibles si los pueblos eran capaces de comprenderlas y recibían el apoyo de todas las clases de la sociedad (Magariños Cervantes, 1858a).

\section{DE SUSCRIPTORES Y LECTORES DE LA BIBLIOTECA}

Una y otra vez a lo largo de la "Crónica de la Biblioteca", el editor señala que eran los suscriptores quienes hacían posible el proyecto, estimando que

para que la Biblioteca pueda, no ya compensar el trabajo que ocasiona, sino para hacer frente a sus gastos de impresión, administración, correspondencia, transporte etc. etc.; para que pueda anticipar erogaciones considerables, popularizarse, mejorar su parte material, marchar con toda regularidad, y ofrecer a los autores un estímulo o compensación, que contribuya a vencer el desencanto que se ha apoderado de la mayor parte de ellos, ante el coloso de la indiferencia publica, la Biblioteca necesita por lo menos quinientos suscriptores fijos solo en Buenos Aires y en Montevideo (Magariños Cervantes, 1858b, pp. 308).

También es allí donde comparte las listas de suscriptores con las que

el curioso lector puede ejercitarse en la aritmética (a la vez que) los individuos, cuyos nombres falten ó estén equivocados, se sirvan avisar, si quieren, en el punto donde se hallan suscripto, y oportunamente se salvará la omisión ó el error en las listas sucesivas (Cané, 1858, p. 273-277).g

Figura 6. Suscriptores

LISTAS DE SUSCRICION.

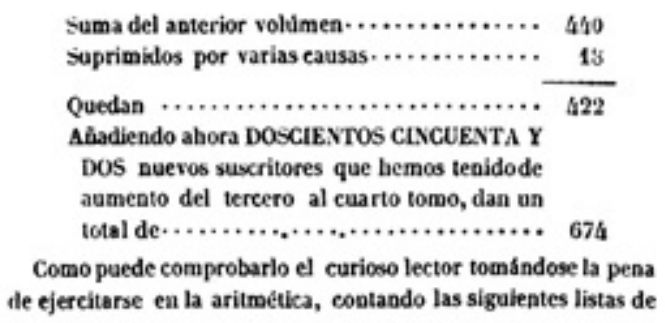

En tres de las publicaciones aparecen listas de suscriptores: Horas de melancolía, donde además se aclaraban las condiciones de la suscripción, No hay mal que por bien no venga y Esther. Este listado, presente en las publicaciones del siglo XIX y que perdura durante los primeros años del siguiente, fue una práctica que no estuvo exenta de críticas, indicándose que solo servía para gastar folios. Pero también permitía descubrir a los lectores. Si bien el propósito de la Biblioteca Americana no era la búsqueda de ganancias, Magariños Cervantes no dejaba de ver el negocio detrás de la edición de libros, reconociendo que el verdadero progreso editorial no se media por el número de suscriptores, sino por la utilidad que dejaban. Estas empresas no llegaban a buen término con hermosas frases e hipótesis ya que en este comercio dos y dos no siempre eran cuatro y no era oro todo lo que reluce (Cané, 1858). 
Tal es así que Magariños Cervantes señaló, guiado por su experiencia, que quemaría los libros antes que tener que cederlos para que se vendan como papel viejo. Como editor, prescindiendo de una eventual ganancia, tomó por única regla el número de suscriptores, mandando a imprimir mil ejemplares, aun sabiendo que de ese número un diez por ciento estaría perdido por distintos siniestros como extravío de ejemplares, suscripciones impagas, roturas o giros de importes no realizados. Sin dudas, la distribución era una operación compleja, sobre todo al tomar en cuenta que la colección llegaba a Paraguay, la República Oriental, la Confederación y el Estado de Buenos Aires y que en estos circuitos no todos contaban con libreros y corresponsales que cumplieran debidamente sus deberes de empadronar a los interesados, cobrar contra entrega del libro, enviar el dinero y devolver los sobrantes (Cané, 1858).

Sin embargo, hay indicios que permiten descubrir a los lectores tras las suscripciones. "Mis lectores, y sobre todo, mis lectoras me lo dirán", afirma Magariños Cervantes en Horas de melancolía (1858, p. 30). Si bien en las listas publicadas el nombre de mujeres es muy pequeño: en la Confederación Argentina se encuentra a Rosario Albornos, en Tala; en Buenos Aires a Catalina Demarchi, Juliana Gauna y Dominga Villoldo de Díaz, en Bragado; destaca el caso de Juana Barreyro de Meyrelles de quien, además de informarse que vivía en Exaltación de la Cruz, se señalaba su profesión: preceptora (Cané, 1858).

Este dato, en apariencia menor, amplía los usos del libro mostrando otros posibles lugares de lectura. Así la suscripción no solo permitía incrementar o armar una biblioteca en el hogar sino que daba cuenta de que los libros también podrían ser utilizados en otros espacios y con otros propósitos. ¿Se usaban entonces estas colecciones para la enseñanza y el aprendizaje?

Michel De Certeau (2006) dice que cada historiador sigue siendo un poeta del detalle y hace sonar sin cesar, como el esteta, las mil armonías que una pieza rara despierta en un campo de conocimientos, entendiendo que el detalle es el que hace la excepción. Así, al volver al catálogo y prestar atención al detalle destaca El Tempe argentino de Marcos Sastre. Esta obra, como otras presentes en la colección, no había sido pensada de una sola vez sino que se había compuesto a partir de artículos que habían aparecido publicados en el Nacional y en $L a$ Gaceta de Buenos Aires. ¿Se había comprado la Biblioteca Americana porque presentaba un fácil acceso a esos materiales? O, ¿será que las mujeres también veían en estos libros un patrimonio que les permitía intervenir en el espacio público?

La idea de que estos libros formaron a hombres y mujeres se ve reforzada con la noticia de que el Gobierno y la Municipalidad de Buenos Aires destinaron cuatrocientos pesos fuertes para comprar varios ejemplares de este tomo, "decretado su adopción como texto de lectura" a la vez que se los distribuiría como premios en las escuelas del Estado. También se informó que dado que la edición estaba agotada, solo se habían podido poner a disposición del Departamento de Escuelas quince o veinte tomos, por lo que el autor realizaría otra edición por su cuenta (Varela, 1859).

Así el proyecto de la Biblioteca era acompañado por prácticas que muestran que el emprendimiento editorial necesitaba de la contribución de autores y editores de varias maneras: ofrecer las obrar a publicar, no obtener ganancias y brindar al público nuevas ediciones de los libros por su cuenta y riesgo. También se entendía que hacer esto colaboraba con la difusión del proyecto de la Biblioteca Americana, afirmándose que cuando el público se acostumbrara a las producciones del genio americano, este se generalizaría, lo cual dándole forma a un círculo virtuoso terminaría por incrementar el número de los suscriptores (Gutiérrez, 1859). Estos suscriptores no solo importaban porque hacían posible el emprendimiento editorial (que en este caso contaba con más de novecientos según se informó), sino porque el editor encontraba este número considerable y promisorio. Señalaba que, si eran ciertos los cálculos del bibliófilo y bibliotecario Charles Nodier, por cada suscriptor al menos seis personas leían un libro gratis (Gutiérrez, 1859), lo que en el futuro podría llevar a aumentar el número de quienes quisieran adquirir los libros.

La favorable acogida que obtuvo esa publicación, que al suspenderse por los sucesos de la guerra, contaba con novecientos suscritores en la República Oriental, Estado de Buenos Aires, Confederación y Paraguay, según consta de las listas publicadas 
al fin de cada tomo, me hizo creer en la posibilidad de que alcanzase larga vida, y más de una vez he ideado diversas combinaciones para llegar a ese resultado (Gutiérrez, 1860, p. 9).

Sin embargo, con Los apuntes biográficos... de Gutiérrez se anunciaba el adiós de la publicación de la Biblioteca Americana, señalando que este último tomo solo podía ofrecerse debido al desinterés y buena voluntad de Carlos Casavalle, quien lo imprimió asumiendo todos los costos de edición y distribución. Magariños Cervantes se despedía

esperando que si no nos es dado más adelante llevar a feliz término la obra emprendida, otros habrá más capaces que, en época más propicia, sabrán fecundizar la idea en germen que nosotros a pesar de todos nuestros esfuerzos no hemos logrado ni lograremos tal vez desarrollar. Entre tanto como la esperanza es lo último que abandona al hombre, confiamos todavía, y estrechando la mano á nuestros amigos, les decimos únicamente: ADIÓS! (Gutiérrez, 1860, p. 6).

\section{CONCLUSIÓN O LA IMPORTANCIA DEL DETALLE}

Al considerar las palabras de De Certeau (2006) y prestar atención al detalle, la Biblioteca Americana de Magariños Cervantes apareció como una pieza rara que muestra al editor en acción, imaginando y materializando un catálogo, al que se va dando forma ajustándolo a las circunstancias que le dificultaban llevar adelante dicha empresa. Esta colección poco parece tener en común con los contenidos del proyecto que lo había inspirado, el de Bello y García del Río, y aun cuando se la pensó como una lección que no debía ser olvidada, las mismas dificultades limitaron su desarrollo y otras más lo complicaron: pocos talleres de impresión, problemas con el papel, limitadas redes de distribución, problemas para el cobro de las suscripciones, falta de financiamiento y demoras en la entrega de los textos. Si bien muchas de estas cuestiones todavía tienen que ser estudiadas, pensar en las suscripciones posibilita el análisis de dos aspectos esenciales de este proyecto: el catálogo y los lectores.

En cuanto al primero, se encuentra que el editor había ideado una colección con dos series: una rioplatense, de la cual brinda cerca de unos cincuenta nombres y otra que incluiría autores del resto de América. Según las características que las definían se tendría un libro por mes, de unas 300 páginas, con cuidada impresión destinados a difundir material inédito de autores americanos. Hasta aquí la idea. Luego los límites. La mayoría de esos libros nunca llegaron a materializarse, aun cuando se había anunciado que estaban en prensa o que los autores habían solicitado tiempo extra para ordenar sus papeles o terminar sus nuevas obras.

Aun con diferentes estrategias, tales como pensar en reediciones, cambiar las obras, alterar el orden de aparición de los autores, dejar de anunciar los próximos títulos o que el autor o el impresor editen los libros por su cuenta y riesgo, fue imposible cumplir con lo anunciado y menos con lo pensado. El derrotero fue complejo; después de anunciar la colección, el primer volumen fue publicado en París en 1854, y cuatro años más tarde, cuando la Biblioteca Americana pasó a imprimirse en Buenos Aires, el proyecto volvió a ponerse en marcha con la promesa de un libro por mes para el segundo semestre de ese año. Tampoco pudo cumplirse con eso y solo un título coincidió con lo anunciado en prensa: No hay mal que por bien no venga del propio Magariños Cervantes y, aunque ese año se suman tres volúmenes a la colección, no se llegó a satisfacer lo prometido a los suscriptores.

A esos cinco tomos, dos años más tarde -con el tomo VII publicado después del tomo VIII-se anunció el fin de la Biblioteca. Pero fue un fin provisorio porque a diez años de la aparición del primer tomo, en 1864 se publicó otro libro que se solicita sea considerado como parte de la colección aun cuando no se mantiene el formato. Este debió ajustarse por la falta de papel, apareciendo en un tomo lo que debía publicarse en dos (aunque solo contaba con 256 páginas). Además se le había aumentado el precio de la suscripción.

Esos diez tomos de la Biblioteca Americana publicados con el objeto de fomentar, difundir y popularizar las obras de autores americanos también permitieron, desde los suscriptores llegar a los lectores. No solo aquellos que se reconocían entre los nombres de los más importantes miembros de la sociedad política, económica, social y cultural sino esos otros hombres y mujeres anónimos que permiten reconstruir otras prácticas. Como 
aquellas que llevan a pensar en la posibilidad de que la Biblioteca haya sido comprada, no para el uso en el hogar, sino como material para enseñar y aprender. Es decir no solo porque el Estado incorporaba alguno de sus tomos como material obligatorio en las escuelas, sino porque las maestras la pensaban como una lectura que podía ser incluida en sus clases. También habla de la existencia de otras prácticas vinculadas al libro como el préstamo entre amigos y conocidos, sobre todo a partir de las estimaciones que señalaban que cada volumen era leído por seis personas.

Estos libros cuentan una pequeña historia. De cuando editar era una tarea que se sumaba a la lista de muchas ocupaciones; cuando los autores no eran escritores a tiempo completo y la búsqueda de ganancia no era el objetivo principal al desarrollar esta actividad. Estos libros comienzan a descubrir a los lectores. No solo porque eran los que hacían viable el proyecto sino porque muestran indicios de prácticas asociadas al libro y la lectura. Bibliotecas para el hogar, bibliotecas para el aula. Que las imprentas se hayan visto desbordadas hablaba de la necesidad de más talleres, cajistas y encuadernadores; es decir mostraba los primeros pasos en la conformación de lo que llegaría a ser la industria editorial. Pero también daban cuenta de unas sociedades que leían. Que leían mucho, en Buenos Aires, en Montevideo y en el interior.

Un editor, una colección... ¿Cuánto permite estudiar la historia de la edición y la historia de la lectura a mediados del siglo XIX? En primera persona, las "Crónicas de una Biblioteca" presentan el recorrido de una idea y los problemas para materializarla. Con los libros también se descubre el mundo de los suscriptores y tras ellos los lectores que, aunque de una manera indirecta y fragmentaria, dan cuenta de sus prácticas. Queda mucho por descubrir en la vida de los libros, pero las herramientas de la microhistoria ayudan a comenzar este recorrido y diseñar nuevas estrategias para su abordaje. Y estos son los detalles que parecen indicar que pueden cumplirse las esperanzas de Magariños Cervantes: "es de grande importancia para la apreciación de los hombres, y para la futura historia de la literatura americana". Comenzamos a entrar en materia siguiendo la ruta de la excepción.

\section{FUENTES DOCUMENTALES}

Bello, A. (1984). Epistolario. Obras completas (vol. XXV). Caracas: Fundación La Casa de Bello.

Biblioteca Americana o Miscelánea de Literatura, artes i ciencias. (1823). Londres: G. Marchant, IngramCourt.

Cané, M. (p). (1858). Esther. Novela original. Buenos Aires: Imprenta de Mayo.

Epistola-Programa de la Biblioteca Americana. Carta de Alejandro Magariños Cervantes a Don Juan Bautista París, 10 de Marzo de 1854. París. Imprenta de los Señores Bernard y Cia.

Gutiérrez, J. M. (1859). Pensamientos, máximas y sentencias de escritores, oradores y hombres de Estado de la República Argentina. Buenos Aires: Imprenta de Mayo.

Gutiérrez, J. M. (1860). Apuntes Biográficos de Escritores, Oradores y hombres de Estado de la República Argentina. Buenos Aires: Imprenta de Mayo.

Hartzenbusch e Hiriart, E. (1894). Apuntes para un catálogo de periódicos madrileños desde el año de 1661 al 1870. Madrid: Sucesores de Rivadeneyra.

Magariños Cervantes, A. (1858a). Horas de melancolía. Buenos Aires: Imprenta de Mayo.

Magariños Cervantes, A. (1858b) No hay mal que por bien no venga. Buenos Aires: Imprenta de Mayo.

Magariños Cervantes, A. (1864). Brisas del Plata. Montevideo: Imprenta tipográfica a vapor.

Mitre, B. (1889). Arengas de Bartolomé Mitre; colección de discursos parlamentarios, politicos, económicos $y$ literarios, oraciones fúnebres, alocuciones conmemorativas, proclamas y alegatos "in voce" pronunciados desde 1848 hasta 1888. Buenos Aires: Librería de Mayo.

Varela, F. (1859). Escritos politicos, económicos y literarios. Buenos Aires: Imprenta del Orden. 


\section{REFERENCIAS}

Amunátegui Aldunate, M. L. (1882). Vida de Andrés Bello. Santiago de Chile: P.G. Ramirez Impresor.

Auza, N. (2002). Alejandro Magariños Cervantes: La Revista Española de ambos mundos y la Biblioteca Americana. Buenos Aires: El Galeón.

Coll, M. (2018). Lexicografía en clave literaria. El caso de Alejandro Magariños Cervantes. Nueva revista de filología hispánica, 66(1), 43-70. Recuperado de https://dx.doi.org/10.24201/nrfh.v66i1.3392

Coronado, M. (1885) Literatura americana (vols. I y II). Buenos Aires: Igón Hermanos.

Corpancho, M. N. (1863) Flores Del Nuevo Mundo: Tesoro Del Parnaso Americano: Compilación de poesías liricas de autores del presente siglo, procedida de un discurso preliminar sobre la poesía lírica en la América latina y arreglada y escogida. México: Vicente García Torres.

Cortés, J. D. (1875) Poesias selectas americanas: con noticias biográficas de los autores: América poética. Paris: Librería de A. Bouret e hijo.

Curia, B. (2012). El primer novelista argentino: Miguel Cané (padre), 1812-1863. Buenos Aires: Editorial Teseo.

De Certeau, M. (2006). La escritura de la historia. México: Universidad Iberoamericana.

de Mendive, R. M. y García, J. J. (1854) América poética: colección de las mejores composiciones escritas por los poetas hispano-americanos del siglo actual (vol. I). La Habana: Tiempo.

de Mendive, R. M. y García, J. J. (1856) América poética: colección de las mejores composiciones escritas por los poetas hispano-americanos del siglo actual (vol. II). La Habana: Tiempo.

Ginzburg, C. (2004). Tentativas. Rosario: Prohistoria Ediciones.

Ginzburg, C. (2010). El hilo y las huellas: lo verdadero, lo falso, lo ficticio. Buenos Aires: Fondo de Cultura Económica.

Gutiérrez,J. M. (1846). América poética. Colección escogida de composiciones en verso escritas por americanos en el presente siglo. Parte lírica. Valparaíso: Imprenta del Mercurio.

Jackson, H. J. (2001). Marginalia: Readers Writing in Books. Yale University Press.

Jaksic Andrade, I. (2001). Andrés Bello: la pasión por el orden. Santiago de Chile: Editorial Universitaria.

Lagomaggiore, F. (1890). América Literaria. Buenos Aires: Imprenta de La Nación.

Núñez, E. (1959). Teoría y proceso de la antología. Cuadernos americanos, 18(106), 257-267.

Parada, A. E. (2012). El dédalo y su ovillo: ensayos sobre la palpitante cultura impresa en la Argentina. Buenos Aires: Instituto de Investigaciones Bibliotecológicas, Facultad de Filosofía y Letras, Universidad de Buenos Aires.

Pas, H. F. (2010). La crítica editada. Juan María Gutiérrez y la América poética. Orbis tertius, 15(16). Recuperado de: http://www.memoria.fahce.unlp.edu.ar/art_revistas/pr.4238/pr.4238.pdf

Pivel Devoto, J. E. (1963 [1854]). Prólogo. En A. Magariños Cervantes, Estudios históricos, politicos y sociales sobre el Rio de la Plata (pp. 35-36). Montevideo: Biblioteca Artigas-Ministerio de Instrucción Pública.

Romagosa, C. (1897). Joyas poéticas americanas. Colección de poesías escogidas originales de autores nacidos en América. Córdoba: Imprenta La Minerva.

Stoddard, R. E. (1985). Marks in books. Cambridge, Mass: Havard University, Houghton Library.

\section{Notas}

1 Estas indagaciones que partieron del estudio de la digitalización de la colección de libros del siglo XIX de la Biblioteca de la Academia Argentina de Letras ha pasado a formar parte de un trabajo que se inscribe en el proyecto de investigación FILO: CyT, Editar y leer en la Universidad. Una historia de la edición académica de la Facultad de Filosofía y Letras (UBA) desde la historia de la lectura. Al rastrear los ejemplares del libro de Miguel Cané (p) se encontró que varios de ellos forman parte de la colección de la Biblioteca Central "Prof. Augusto Cortázar", lo que permite analizar otros aspectos de estos libros y permitirá ampliar la investigación a los objetivos del proyecto, respondiendo a cuestiones relacionadas con cómo fue que llegaron esos libros a formar parte del acervo de la Biblioteca, cuáles fueron sus usos: si eran consultados en sala o fueron incorporados estos materiales a los programas de estudios, entre otras cuestiones. 
2 Para reconstruir su vida pueden consultarse la nota autobiográfica que se incluye en el tomo III de la Biblioteca Americana: Magariños Cervantes, A. (1858). No hay mal que por bien no venga. Buenos Aires: Imprenta de Mayo y la noticia biográfica firmada por Heraclio Fajardo incluida en Brisas del Plata. (Magariños Cervantes, A. (1864). Brisas del Plata. Montevideo: Imprenta Tipográfica a Vapor). También estos trabajos: Auza, N. T. (2002). Alejandro Magariños Cervantes: la Revista Española de Ambos Mundos y la Biblioteca Americana. Montevideo: El Galeón; Giraldi, N. (2001). Alejandro Magariños Cervantes. En Oreggioni, A. (Dir). Nuevo diccionario de literatura uruguaya. Montevideo: Ediciones de la Banda Oriental; Pivel Devoto, J. E. (1963 [1854]). "Prólogo" a Estudios históricos, políticos y sociales sobre el Rio de la Plata de Alejandro Magariños Cervantes. Montevideo: Biblioteca Artigas - Ministerio de Instrucción Pública. (Clásicos uruguayos, 35-36). Rama, Á. (1968). 180 años de literatura. Enciclopedia uruguaya. Tomo 2. Montevideo: Editores Reunidos - Arca. Roxlo, C. (1912). Historia crítica de la literatura uruguaya. Tomo 1: Romanticismo. Montevideo: Barreiro y Ramos. Es interesante destacar que, en 1859, Manuel Ovilo y Otero incluye su biografía en el Manual de biografía y bibliografía de los escritores españoles del siglo XIX (París: Rosa y Bouret, 1859, 2 vols.) y que Pivel Devoto referencia los artículos del diario madrileño El Orden, que a fines del siglo XIX, se dedican al autor. (Tomos 35 y 36 de la Colección de Clásicos Uruguayos. Recuperado de: http://www.artigas.org.uy/bibliotecas/cu/index.php) Por último la Biblioteca digital de autores uruguayos tiene una página dedicada a Magariños Cervantes, desde donde es posible acceder a su obra digitalizada: http://anaforas.fic.edu.uy/jspui/handle/123456789/37692

3 Sobre el papel de Andrés Bello en la Biblioteca, con la identificación de autoría de los diversos artículos, véase: Grases, P. (1981). "La Biblioteca Americana. Londres, 1823" en Estudios sobre Andrés Bello, 2 tomos. Caracas - Barcelona México: Seix Barral. Tomo II. 318-328; Grases, P. (1981). “Tres empresas periodísticas de Andrés Bello” en Estudios sobre Andrés Bello, 2 tomos. Caracas - Barcelona - México: Seix Barral. Tomo II. 307-314. De Silva Castro, R. (1973). "Andrés Bello en el periodismo", en Alamiro de Ávila, M. Estudios sobre la vida y obra de Andrés Bello, Santiago: Editorial Universitaria.

4 La América poética instauró el género antología con parámetros típicamente modernos, entre los cuales debe destacarse la mediación del juicio crítico como fundamento del recorte propuesto. Ya desde el Prospecto aparece la función del editor al señalar que para la elección de las piezas se ha tomado como guía el amor discreto por el nombre americano y los consejos inmutables del buen gusto (Pas, 2010).

\section{BY-NC-SA}

